\title{
A Radar Cross-Section Model for Power Lines at Millimeter-Wave Frequencies
}

\author{
Kamal Sarabandi, Fellow, IEEE, and Moonsoo Park
}

\begin{abstract}
The knowledge of radar backscatter characteristics of high-voltage power lines is of great importance in the development of a millimeter-wave wire detection system. In this paper, a very high-frequency technique based on an iterative physical optics approach is developed for predicting polarimetric radar backscattering behavior of power lines of arbitrary strand arrangement. In the proposed scattering model the induced surface current is obtained using the tangent plane approximation in an iterative manner where the first-order current, obtained from the incident wave, is used as the source for the second-order current and so on. The approximation is valid for frequencies where the cable strand diameter is on the order of or larger than the wavelength. It is shown that the copolarized backscatter is dominated by the contribution from the first-order PO currents, whereas the cross-polarized backscatter is generated by the second- and higher order PO currents. Using this model, the effects of radar antenna footprint, surface irregularities, and cable sag (when suspended between towers) on radar backscatter are studied. To verify the validity of the proposed model, theoretical results are compared at $94 \mathrm{GHz}$ with experimental results and are found to be in good agreement.
\end{abstract}

Index Terms-Iterative solution, numerical analysis, physical optics, power line, radar cross-section (RCS).

\section{INTRODUCTION}

$\mathbf{H}$ IGH-VOLTAGE power lines and their supporting towers are considered as a major safety hazard for helicopters and other low-flying aircrafts. Because of their low observability, in the United States alone, hundreds of helicopter collisions with power lines are reported [1]. To prevent these accidents, development of small size, low cost, millimeter-wave collision avoidance radar system is being considered. In a recent study [2], we proposed a detection algorithm that makes use of polarimetric backscatter statistics of distributed targets and power lines at $W$ band frequencies. To examine the performance of such algorithms, a thorough knowledge of scattering behavior of different power lines with different structural parameters such as strand arrangements and strand diameter is needed. Such comprehensive radar cross-section (RCS) characterization experimentally seems very difficult if not impossible. The literature concerning analytical evaluation of RCS of power lines is limited to simple models proposed by Rembold [3] and Al-Khatib [4]. Rembold used a long wire

Manuscript received February 3, 1999; revised May 2, 2001. This work was supported by the U.S. Army Research Laboratory under Cooperative Agreement DAAL01-96-2-001 through collaborative participation in the Advanced Sensors Consortium.

The authors are with the Radiation Laboratory, Department of Electrical Engineering and Computer Science, The University of Michigan, Ann Arbor, MI 48109-2122 USA (e-mail: saraband@eecs.umich.edu).

Digital Object Identifier 10.1109/TAP.2003.816380 (smooth cylinder) model, which is valid only at microwave frequencies ( $X$ band and below). On the other hand, the model developed by Al-Khatib is a very high-frequency model based on geometrical optics (GO) approximation. Unlike Rembold's model the GO model is able to account for the effect of periodic structure of power lines. Using this model, Al-Khatib was able to predict the existence and locations of Bragg backscatter generated by the power line structure. Due to the fundamental limitations in GO approximation, this model is incapable of predicting the cross-polarized backscatter and differentiating the possible differences that may exist between the copolarized backscatter ( $S_{v v}$ and $S_{h h}$ ) responses. Also in [5] measurements of infrared reflectance from power lines are reported. Based on the model developed here and the phenomenological study reported in [2], detection of power lines in polarimetric SAR images at $K a$ band was demonstrated recently [6].

Because of its simplicity, physical optics (PO) model is widely used for the analysis of RCS of complex targets at high frequencies. Like GO, PO approximation is valid at high frequencies and cannot predict the cross-polarized backscatter for perfectly conducting targets. However, the concept of tangent plane approximation used in the PO approximation may be employed repeatedly to get more accurate results. The induced electric current on the surface of a perfectly electric conducting (PEC) body is proportional to the total tangential magnetic field. In the PO approach, the induced current is approximated by $\mathbf{J}_{p o}=2\left(\hat{n} \times \mathbf{H}_{i}\right)$, where $\mathbf{H}_{i}$ is the incident magnetic field. The field produced by this current does not include the effect of multiple scattering, which gives rise to cross-polarized backscatter. Experimental results show [2], [7] that at high frequencies ( $W$ band and higher) the effect of multiple scattering on the surface of power lines becomes significant. The so-called multiple scattering is caused by the periodic helical arrangement of wire strands. From a simple geometrical optics point of view the existence of cross-polarized backscatter caused by multiple scattering can be attributed to the tilted dihedral shape grooves that exist between adjacent wire strands. Accurate polarimetric backscatter behavior of power line structure can be determined by resorting to numerical full-wave analysis. In Section II, an integral equation for the induced surface current density is derived that makes use of a periodic dyadic Green's function. This integral equation is than used as a basis for the iterative PO model. In Section III, the formulation for the iterative PO model is described where the traditional PO current is used as a source of radiation for computing the second-order PO current on the surface. The iterative PO model allows for prediction of angular RCS response of power lines when illuminated by plane waves and spherical waves. Numerical simulations and comparison 
with measurements at $W$ band frequencies are presented in Section IV. Also the effect of irregularities on the periodic arrangements of strands on the RCS is examined.

\section{FORMULATION OF DYADIC GREEN'S FUNCTION FOR POWER LINES}

The geometry of a power line and its associated coordinate system is shown in Fig. 1. Let us consider a plane wave propagating along $\hat{k}_{i}=k_{x i} \hat{x}+k_{y i} \hat{y}+k_{z i} \hat{z}$ direction whose electric field is represented by

$$
\mathbf{E}^{i}=\hat{p} e^{i k_{0} \hat{k}_{i} \cdot \mathbf{r}}
$$

where $\hat{p}$ denotes the polarization vector. Since the incident field is plane wave and the surface of the power line is periodic along the $z$-axis, the induced surface electric current distribution is also periodic except for a progressive phase factor $e^{i k_{0} k_{i z} z}$. Hence the current on the $n$th period can be expressed in terms of the current on the period near the origin as

$$
\mathbf{J}\left(\mathbf{r}_{n}^{\prime}\right)=\mathbf{J}\left(\mathbf{r}_{0}^{\prime}\right) e^{i k_{0} k_{i z} n L}
$$

where $L$ is the period and

$$
\mathbf{r}_{n}^{\prime}=\mathbf{r}_{0}^{\prime}+n L \hat{z} .
$$

Subdividing the surface of the scatterer into an infinite number segments each having a length $L$, the total scattered field can be computed using the free-space Green's function and is given by

$$
\mathbf{E}^{s}(\mathbf{r})=i k_{0} Z_{0} \sum_{n=-\infty}^{+\infty} \int_{S_{n}} \overline{\bar{G}}\left(\mathbf{r}, \mathbf{r}_{n}^{\prime}\right) \cdot \mathbf{J}\left(\mathbf{r}_{n}^{\prime}\right) d \mathbf{r}_{n}^{\prime}
$$

where $\mathbf{r}_{n}^{\prime} \in S_{n}$ and $Z_{0}$ is the free-space intrinsic impedance. By inserting (2) and (3) into (4), and interchanging the order of integration and summation, (4) can be rewritten as

$\mathbf{E}^{s}(\mathbf{r})=i k_{0} Z_{0} \int_{S_{0}}\left[\sum_{n=-\infty}^{+\infty} \overline{\bar{G}}\left(\mathbf{r}, \mathbf{r}_{0}^{\prime}+n L \hat{z}\right) e^{i k_{0} k_{i z} n L}\right] \cdot \mathbf{J}\left(\mathbf{r}_{0}^{\prime}\right) d \mathbf{r}_{0}^{\prime}$.

The quantity inside the bracket is the dyadic Green's function for periodic structures and is given by

$$
\overline{\bar{G}}_{p}\left(\mathbf{r}, \mathbf{r}_{0}^{\prime}\right)=\left(\overline{\bar{I}}+\frac{1}{k_{0}^{2}} \nabla \nabla\right) \sum_{n=-\infty}^{+\infty} g\left(\mathbf{r}, \mathbf{r}_{0}^{\prime}+n L \hat{z}\right) e^{i k_{0} k_{i z} n L}
$$

where $g\left(\mathbf{r}, \mathbf{r}^{\prime}\right)$ is the free-space scalar Green's function given by

$$
g\left(\mathbf{r}, \mathbf{r}^{\prime}\right)=\frac{1}{4 \pi} \frac{e^{i k_{0}\left|\mathbf{r}-\mathbf{r}^{\prime}\right|}}{\left|\mathbf{r}-\mathbf{r}^{\prime}\right|}
$$

Expressing $g\left(\mathbf{r}, \mathbf{r}^{\prime}\right)$ in terms of its two-dimensional Fourier expansion, invoking Poisson summation formula, and some other algebraic manipulations, the periodic Green's function is found to be

$$
\overline{\bar{G}}_{p}\left(\mathbf{r}, \mathbf{r}^{\prime}\right)=\left(\overline{\bar{I}}+\frac{1}{k_{0}^{2}} \nabla \nabla\right) g_{p}\left(\mathbf{r}, \mathbf{r}^{\prime}\right)
$$

where

$$
\begin{aligned}
g_{p}\left(\mathbf{r}, \mathbf{r}^{\prime}\right)= & \frac{i}{4 L} \sum_{n=-\infty}^{+\infty} H_{0}^{(1)}\left(k_{0} \sin \theta_{n}\left|\vec{\rho}-\vec{\rho}^{\prime}\right|\right) \\
& \times e^{i k_{0} \cos \theta_{n}\left(z-z^{\prime}\right)} . \\
\sin \theta_{n}= & \sqrt{1-\left(\frac{n \lambda}{L}+k_{i z}\right)^{2}} .
\end{aligned}
$$

The summation in (9) converges very rapidly as the argument of the Hankel function becomes pure imaginary for large values of $n$. The scattered magnetic field can be obtained from (5) by taking curl of the electric field. Also noting that $\nabla \times \overline{\bar{G}}_{p}\left(\mathbf{r}, \mathbf{r}^{\prime}\right)=$ $\nabla g_{p}\left(\mathbf{r}, \mathbf{r}^{\prime}\right) \times \overline{\bar{I}}$ and the induced electric current is proportional to the total tangential magnetic field, i.e., $\mathbf{J}(\mathbf{r})=\hat{n} \times\left(\mathbf{H}^{i}+\mathbf{H}^{s}\right)$, a magnetic field integral equation for the current can be obtained and is given by

$$
\begin{gathered}
\mathbf{J}(\mathbf{r})=2\left(\hat{n}(\mathbf{r}) \times \mathbf{H}^{i}(\mathbf{r})\right)-2 f_{S_{0}}\left[\left(\hat{n}(\mathbf{r}) \cdot \nabla g_{p}\left(\mathbf{r}, \mathbf{r}^{\prime}\right)\right) \mathbf{J}\left(\mathbf{r}^{\prime}\right)\right. \\
-\left(\hat{n}(\mathbf{r}) \cdot \mathbf{J}\left(\mathbf{r}^{\prime}\right) \nabla g_{p}\left(\mathbf{r}, \mathbf{r}^{\prime}\right)\right] d \mathbf{r}^{\prime}
\end{gathered}
$$

where $S_{0}$ specifies the surface of power line over one period and $f_{S_{0}}$ denotes the principal value integral.

Integral equation (10) can be solved numerically for the induced electrical current density $\mathbf{J}(\mathbf{r})$ using the standard method of moments (MOM). Once $\mathbf{J}(\mathbf{r})$ is determined the scattered electric field in the far-field region can be obtained from

$$
\mathbf{E}^{s}(\mathbf{r})=i k_{0} Z_{0} \int_{S_{0}} \overline{\bar{G}}_{p f}\left(\mathbf{r}, \mathbf{r}^{\prime}\right) \cdot \mathbf{J}\left(\mathbf{r}^{\prime}\right) d \mathbf{r}^{\prime}
$$

where $\overline{\bar{G}}_{p f}\left(\mathbf{r}, \mathbf{r}^{\prime}\right)$ is the far-field approximation of the periodic Green's function given by (8). The explicit expression for $\overline{\bar{G}}_{p f}\left(\mathbf{r}, \mathbf{r}^{\prime}\right)$ can be obtained by noting that $\nabla=\nabla_{t}+i k_{0} \cos \theta_{n} \hat{z}$ and the fact that in far-field $\nabla_{t} \simeq i k_{0} \sin \theta_{n} \hat{k}_{\perp}^{s}$ where

$$
\hat{k}_{\perp}^{s}=\cos \varphi_{s} \hat{x}+\sin \varphi_{s} \hat{y}
$$

denotes the direction of observation point. Hence

$$
\begin{aligned}
\overline{\bar{G}}_{p f}\left(\mathbf{r}, \mathbf{r}^{\prime}\right)= & \frac{i}{4 L} \sum_{n \in N}\left[\overline{\bar{I}}-\sin ^{2} \theta_{n} \hat{k}_{\perp}^{s} \hat{k}_{\perp}^{s}-\sin \theta_{n} \cos \theta_{n}\right. \\
& \left.\times\left(\hat{k}_{\perp}^{s} \hat{z}+\hat{z} \hat{k}_{\perp}^{s}\right)-\cos ^{2} \theta_{n} \hat{z} \hat{z}\right] \\
& \cdot H_{0}^{(1)}\left(k_{0} \sin \theta_{n} \rho\right) e^{i k_{0} \cos \theta_{n} z} \\
& \times e^{-i k_{0}\left(\sin \theta_{n}{\overrightarrow{\rho^{\prime}}}^{\prime} \hat{k}_{s}+\cos \theta_{n} z^{\prime}\right)}
\end{aligned}
$$

where $N$ denotes a set of integers for which $\sin \theta_{n}$ is real (these denote the observable bistatic Bragg directions). It should be emphasized that the far-field approximation (11) is valid so long as $\sin \theta_{n} \neq 0$.

\section{PhysicAl OPTICS MODEL}

At low frequencies where typical dimensions of scatterers are comparable to or less than a wavelength, the integral equation given by (10) can be used to obtain an exact solution using the method of moments. But power lines are electrically large at 94 


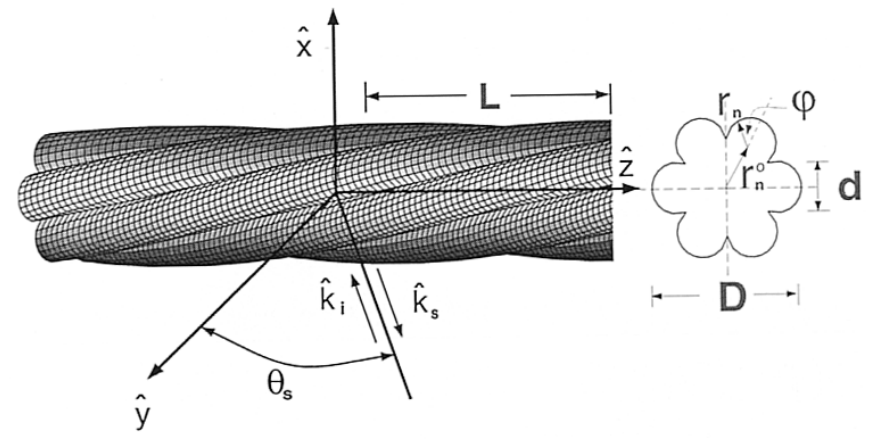

Fig. 1. Geometry of a power line and its important parameters. Here $L$ denotes the surface period, $d$ is the strand diameter, $D$ is the cable diameter, $P$ is the pitch of the helix, and $\hat{k}_{i}$ and $\hat{k}_{s}$ denote the directions of incidence and backscatter.

$\mathrm{GHz}$ and the numerical method would require exorbitant computer memory and computation time. For example, a power line with an overall diameter of $3.5 \mathrm{~cm}$, surface periodicity of 2.4 $\mathrm{cm}$, and strand diameter of $0.446 \mathrm{~cm}$ must be discretized into approximately $5 \times 10^{4}$ small patches, which results in $2 \times 10^{5}$ unknowns (real and imaginary parts of two unknown surface current density) at $94 \mathrm{GHz}$. Although implementation of MOM for this example is not impossible with modern computers, the required memory and computation time are rather high. Besides, for studying the effects of nonuniform illumination and surface irregularities where periodic Green's function can no longer be used, the required computation time and computer memory would far exceed the existing computer capabilities. To alleviate this problem while maintaining a high degree of accuracy, an approximate solution based on iterative physical optics is used [8], [9]. The iterative physical optics is applied using both the periodic and free-space Green's function. The periodic Green's function is appropriate for plane wave illumination assuming a perfect surface periodicity. It can also be used for scattered field evaluation when the power line is illuminated by a nonuniform plane wave by expanding the incident field in terms of a continuous spectrum of plane waves and using superposition. The free-space Green's function allows the computation of RCS of power lines with quasi-periodic surface.

Fig. 1 shows the geometry of a typical power line in the principal plane of a radar antenna. As mentioned earlier, a power line is made up of a number of steel strands (placed at the core) and aluminum wire strands with diameter $d$ wound together in a helical fashion. Depending on the number of wires on the outer surface, the overall diameter of the cable $(D)$, and the pitch of the helix $(P)$, the period of the surface $(L)$ can be determined. A mathematical description of the surface is needed for either MOM or iterative physical optics solution. Consider a power line with $N$ strands at the outer surface (see Fig. 1). After some inspections, a point on the surface of this cable may be represented by

$$
\mathbf{r}=\mathbf{r}_{o}^{n}+\mathbf{r}_{n} \quad n=1,2, \ldots, N
$$

where

$$
\mathbf{r}_{o}^{n}=\left(\frac{D-d}{2}\right) \cos \frac{2 \pi n}{N} \hat{x}+\left(\frac{D-d}{2}\right) \sin \frac{2 \pi n}{N} \hat{y}
$$

TABLE I

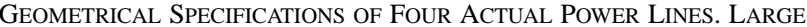
POWER LiNES ARE MADE UP OF ALUMINUM OR COPPER OUTER STRANDS AND SteEl InNer Strands. Here $L$ Denotes the Surface Period, $d$ Is the Strand Diameter, $D$ Is the Cable Diameter, And $P$ Is THE PITCH OF THE HELIX

\begin{tabular}{c||c|c|c|c|c|c|c}
\hline \multicolumn{1}{c||}{ No. } & \multicolumn{2}{|c|}{ Al/Copper } & \multicolumn{2}{c|}{ Steel } & \multirow{2}{*}{ D [cm] } & P [cm] & L [cm] \\
\cline { 2 - 5 } & \# of St. & d [cm] & \# of St. & d [cm] & & & \\
\hline \hline 1 & 7 & 0.400 & - & - & 1.20 & 14.60 & 1.95 \\
\hline 2 & 19 & 0.446 & - & - & 2.22 & 23.5 & 1.52 \\
\hline 3 & 54 & 0.337 & 7 & 0.337 & 3.01 & 35.56 & 2.00 \\
\hline 4 & 45 & 0.446 & 7 & 0.301 & 3.52 & 40.65 & 2.40 \\
\hline
\end{tabular}

$$
\begin{aligned}
\mathbf{r}_{n}= & \left(\frac{d}{2} \cos \frac{2 \pi n}{N} \cos \varphi-\frac{d \sqrt{(\pi D)^{2}+P^{2}}}{2 P}\right. \\
& \left.\quad \times \sin \frac{2 \pi n}{N} \sin \varphi\right) \hat{x} \\
+ & \left(\frac{d}{2} \sin \frac{2 \pi n}{N} \cos \varphi+\frac{d \sqrt{(\pi D)^{2}+P^{2}}}{2 P}\right. \\
& \left.\quad \times \cos \frac{2 \pi n}{N} \sin \varphi\right) \hat{y} .
\end{aligned}
$$

Here, as mentioned before, $d, D$, and $P$ are strand diameter, cable overall diameter, and helix pitch, respectively, and $\varphi$ is a parameter indicating the angle between $\mathbf{r}_{n}$ and $\mathbf{r}_{0}^{n}$. The range of $\varphi$ is limited to $|\varphi|<\varphi_{0}$ where $\varphi_{0}$ is an angle slightly lager than $90^{\circ}$. Finally, the three-dimensional parametric representation of a power line can be represented as

$R=\left[r_{x} \cos \frac{2 \pi z}{P}-r_{y} \sin \frac{2 \pi z}{P}, r_{x} \sin \frac{2 \pi z}{P}+r_{y} \cos \frac{2 \pi z}{P}, z\right]$

where $r_{x}$ and $r_{y}$ are the $x$ and $y$ component of $\mathbf{r}$, respectively. Fig. 1 shows the surface of a power line with six outer strands generated by (16). The starting point in formulating the approximate numerical solution based on PO is the magnetic field integral equation given by (10). In this approximation, the following observations are in order. Most power lines are wound in a manner so that $D / P \ll 1$, and therefore $\hat{n} \cdot \hat{z} \ll 1$. Also at high frequencies where the radius of curvature is larger than the wavelength, the quantity $\hat{n} \cdot\left(\left(\vec{\rho}-\vec{\rho}^{\prime}\right) /\left|\vec{\rho}-\vec{\rho}^{\prime}\right|\right) \ll 1$ for values of $\vec{\rho}^{\prime}$ sufficiently close to $\vec{\rho}$. Hence the principal value integral in (10) is small compared to $\hat{n} \times \mathbf{H}^{i}$.

Table I shows the geometrical parameters of various cables used in power distribution and transmission. To the zeroth order of approximation, the integral in (10) can be ignored, which results in the traditional physical optics current $\left(\mathbf{J}(\mathbf{r}) \simeq \mathbf{J}_{0}(\mathbf{r})=\right.$ $\left.2\left(\hat{n} \times \mathbf{H}^{i}\right)\right)$. Expanding the surface current in terms of a perturbation series and using (10), it can be shown that

$$
\begin{aligned}
\mathbf{J}^{(n)}(\mathbf{r})=-2 f_{S_{0}}[ & \left(\hat{n}(\mathbf{r}) \cdot \nabla g_{p}\left(\mathbf{r}, \mathbf{r}^{\prime}\right)\right) \mathbf{J}^{(n-1)}\left(\mathbf{r}^{\prime}\right) \\
& -\left(\hat{n}(\mathbf{r}) \cdot \mathbf{J}^{(n-1)}\left(\mathbf{r}^{\prime}\right) \nabla g_{p}\left(\mathbf{r}, \mathbf{r}^{\prime}\right)\right] d \mathbf{r}^{\prime}
\end{aligned}
$$

where $\mathbf{J}^{(n)}$ is the $n$ th-order physical optics currents. To study the RCS of power lines for nonuniform illumination and/or 
when the surface is not perfectly periodic, (17) should be modified by replacing $g_{p}\left(\mathbf{r}, \mathbf{r}^{\prime}\right)$ with the free-space scalar Green's function $g\left(\mathbf{r}, \mathbf{r}^{\prime}\right)=(1 / 4 \pi)\left(e^{i k}\left|\mathbf{r}-\mathbf{r}^{\prime}\right| /\left|\mathbf{r}-\mathbf{r}^{\prime}\right|\right)$ and the limit of the integral should be changed to include the entire lit area. The explicit expression for the higher order physical optics current when free-space scalar Green's function is used is given below

$$
\begin{aligned}
\mathbf{J}^{(n)}(\mathbf{r})= & -2 \int_{\text {lit area }}-\left[\hat{n}(\mathbf{r}) \cdot\left(\mathbf{r}-\mathbf{r}^{\prime}\right) \mathbf{J}^{(n-1)}\left(\mathbf{r}^{\prime}\right)-\hat{n}(\mathbf{r})\right. \\
& \left.\cdot \mathbf{J}^{(n-1)}\left(\mathbf{r}^{\prime}\right)\left(\mathbf{r}-\mathbf{r}^{\prime}\right)\right]\left(i k-\frac{1}{\left|\mathbf{r}-\mathbf{r}^{\prime}\right|}\right) \\
& \cdot \frac{e^{i k_{0}\left|\mathbf{r}-\mathbf{r}^{\prime}\right|}}{4 \pi\left|\mathbf{r}-\mathbf{r}^{\prime}\right|^{2}} d \mathbf{r}^{\prime} .
\end{aligned}
$$

Once the PO current to any desired order is obtained, the scattered field in the far-field region can be obtained from

$$
\mathbf{E}^{s}(\mathbf{r})=\frac{i k_{0} Z_{0}}{4 \pi} \frac{e^{i k r}}{r} \int_{\text {litarea }}\left(\overline{\bar{I}}-\hat{k}^{s} \hat{k}^{s}\right) \cdot \mathbf{J}\left(\mathbf{r}^{\prime}\right) e^{-i k_{0} \hat{k}_{s} \cdot \mathbf{r}^{\prime}} d \mathbf{r}^{\prime} .
$$

\section{NUMERICAL AND EXPERIMENTAL RESULTS}

In this section, the validity of the proposed physical optics model is examined by comparing simulated results with backscatter measurements of different power line samples at $94 \mathrm{GHz}$. Also a sensitivity analysis is carried out to study the effects of different geometrical parameters of power lines. The backscatter experiments were conducted on four different power line samples whose parameters are listed in Table I. The detailed experimental procedure and an extensive set of backscatter measurements of different power lines under varieties of physical conditions are reported in a recent paper by the authors [2]. Here we briefly describe the experimental setup. A stepped-frequency radar operating at $94 \mathrm{GHz}$ was used to perform the backscatter measurements at a distance of about $12 \mathrm{~m}$. The half-power beamwidth of the receive antenna is $1.4^{\circ}$, which produces a footprint of about $30 \mathrm{~cm}$ at the target range. The systematic errors in the polarimetric backscatter measurements, which are caused by the antenna crosstalks and channel imbalances, are removed using a calibration procedure that uses two external calibration targets [10]. Since the length of power lines is larger than the extent of antenna footprint, the RCS values reported are functions of the radar parameters. The values reported here are obtained assuming that a power line is a point target located at the antenna boresight. The power line samples were placed in the horizontal plane and their polarimetric backscatter responses were measured in the principal plane of the radar antenna. The angular RCS patterns were obtained over the angular range $-10^{\circ}$ to $50^{\circ}$ with a fine angular resolution $0.1^{\circ}$. Before comparing the analytical results with measured quantities, a sensitivity analysis is carried out to examine the convergence of the iterative PO solution and the effect of the size of the footprint on the backscatter response.

Assuming a plane wave illumination, Fig. 2 shows the contribution to the backscatter of the first- and second-order PO currents for power line \#1. In this case a cable length of $0.3 \mathrm{~m}$ and a center frequency $93.5 \mathrm{GHz}$ are assumed. It is shown that for the copolarized components, the first-order PO solution accurately predicts the backscatter, whereas the dominant source

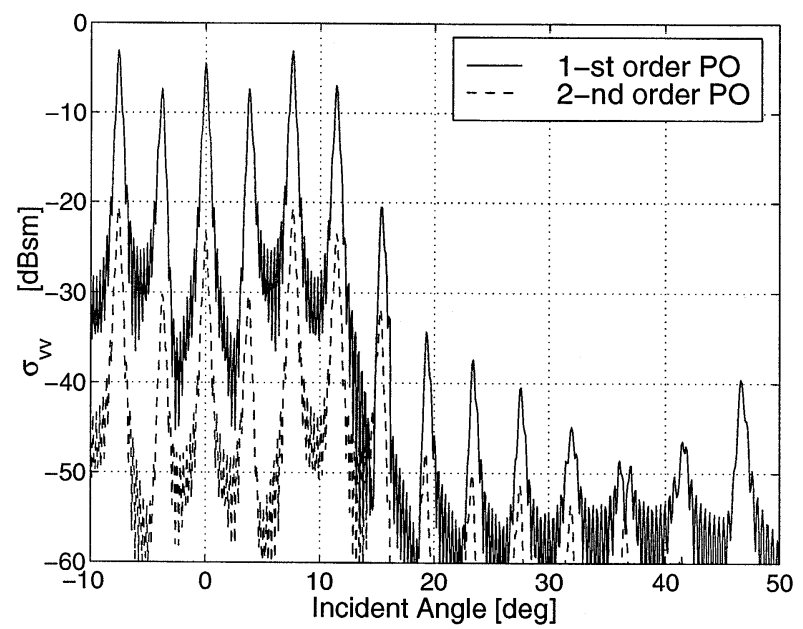

(a)

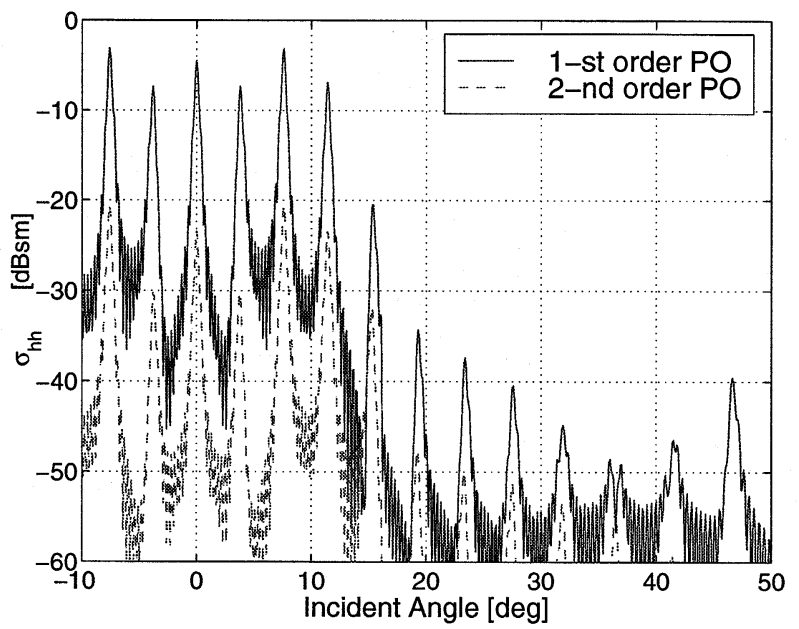

(b)

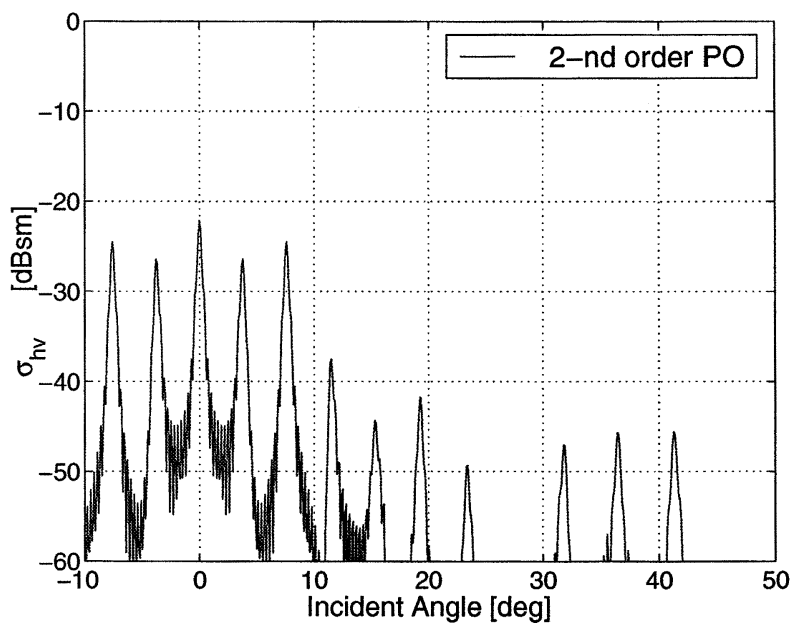

(c)

Fig. 2. RCS of power line \#1 produced by PO. The first- and second-order solutions are shown for the plane wave illumination of a $90-\mathrm{cm}-$ long cable. It is shown that the second-order response is about $15 \mathrm{~dB}$ lower than the first order for $\mathrm{VV}$ and $\mathrm{HH}$. (a) VV polarized response, (b) $\mathrm{HH}$ polarized response, and (c) $\mathrm{HV}$ polarized response.

of cross-polarized component is the second-order PO currents. In fact, the second-order contribution is about $20 \mathrm{~dB}$ below the first order. Similarly, the third order is of the same order of mag- 


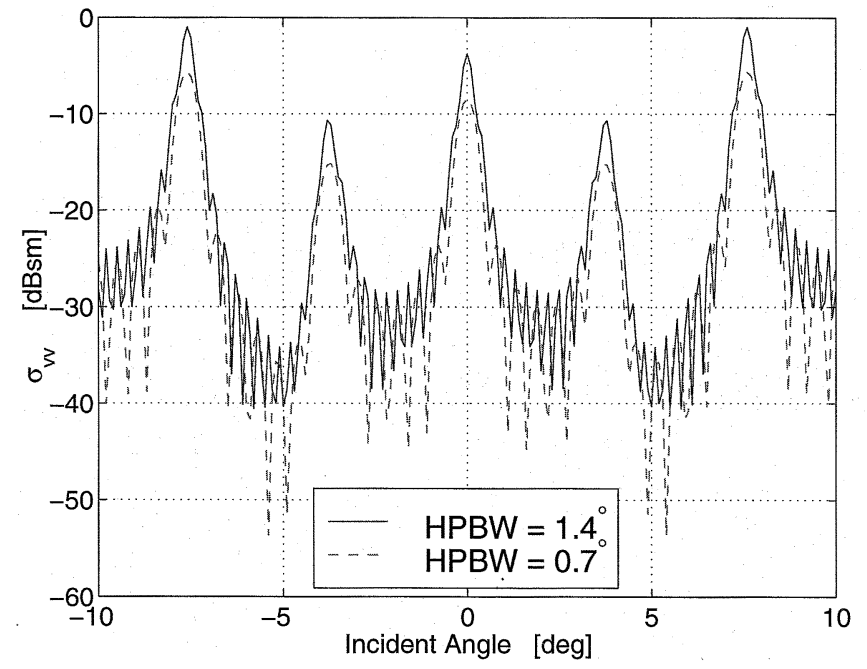

Fig. 3. RCS of cable \#1 when illuminated with an antenna with beamwidth $1.4^{\circ}$ and $0.7^{\circ}$ at a distance $12 \mathrm{~m}$.

nitude smaller than the second order. This also indicates that no higher order currents are needed to predict the polarimetric RCS response of power lines. The direction of significant backscatter readily can be predicted from the periodic dyadic Green's function and is given by

$$
\theta_{n}=\sin ^{-1} \frac{n \lambda}{2 L}
$$

It is shown that levels of backscatter for Bragg modes beyond $15^{\circ}$ incidence are reduced significantly. This is due to the fact that the specular points in backscatter direction cease to exist beyond $15^{\circ}$ incidence. Next we examine the effect of the antenna footprint size on the RCS level and angular pattern. For this simulation we use again the geometry of power line \#1 and consider a radar with Gaussian beam at a distance of $12 \mathrm{~m}$ from the power line. Two cases corresponding to two beamwidths are considered: 1) a half-power beamwidth of $1.4^{\circ}$ corresponding to the beamwidth of the radar used in the experiment and 2) a half-power beamwidth of $0.7^{\circ}$. The lit area for the antenna with beamwidth $0.7^{\circ}$ is half of that of the $1.4^{\circ}$ beamwidth antenna, and therefore we expect the RCS of the former to be $6 \mathrm{~dB}$ lower than the latter.

Fig. 3 compares the VV backscatter response of the power line for the two spherical wave illumination. For these simulations, free-space Green's function formulation was used. It is shown that the RCS pattern for each Bragg mode widens and the peak value drops (more than $6 \mathrm{~dB}$ ), which are caused by the spherical phase front and nonuniform amplitude variations. The same effects are also observed when the RCS of a finite length $(30 \mathrm{~cm})$ cable illuminated by a plane wave is compared with the RCS of the same cable illuminated by the $1.4^{\circ}$ beamwidth antenna. This comparison is shown in Fig. 4 for vertical polarization. Fig.5 shows the comparison between the complete second-order PO model results with those measured using the University of Michigan 94-GHz scatterometer. The overall agreement between calculated and measured co- and cross-polarized backscatter for power line \#1 is very good. The slight discrepancies are mainly attributed to imperfection in the geometry of the power line. The agreement between the measured results and the second-order PO degrades as the number of stands is increased. This issue is addressed next.

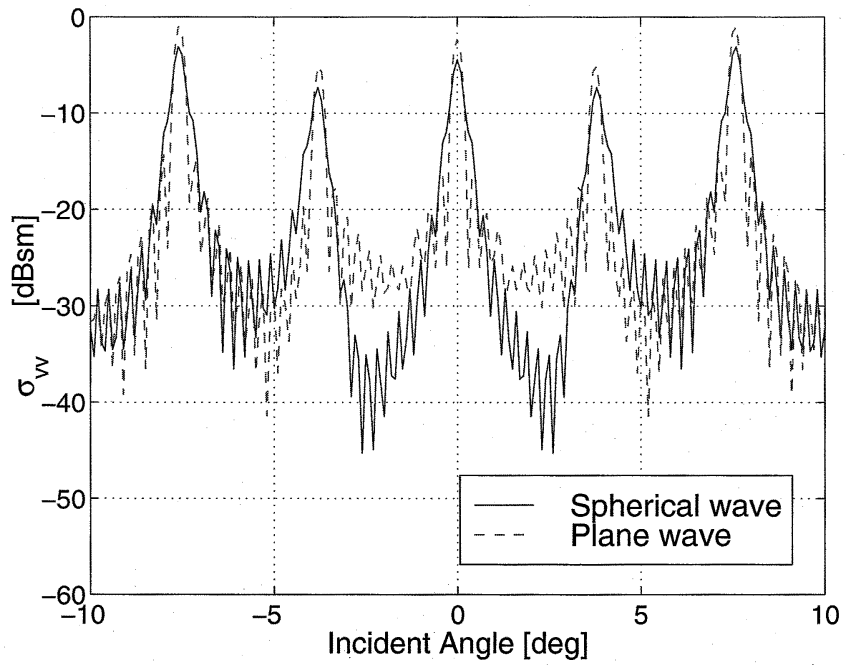

Fig. 4. Comparison of RCS for plane wave illumination over finite length 30 $\mathrm{cm}$ and a spherical wave illumination assuming plane wave incidence observed by an antenna with a Gaussian beam and beamwidth $1.4^{\circ}$ at a range $12 \mathrm{~m}$ from the power line. The $3-\mathrm{dB}$ footprint is $30 \mathrm{~cm}$ in this case. Range $=12(\mathrm{~m})$, Beamwidth $=1.4^{\circ}$

Flexing and straightening of power line cables perturbs the periodic arrangement of the outer strands. At $W$ band frequencies, a movement of outer strands as small as $0.5 \mathrm{~mm}$ can have a significant impact on its RCS pattern. To simulate the effect of surface irregularities a variable gap (spacing) with a mean of about $1 / 20$ of the strand diameter was inserted between adjacent strands randomly. A Gaussian distribution was used to specify the gap width. Fig. 6 shows the RCS patterns of cable \#1 for a perfect periodic and quasi-periodic surface structure. In this case, a nonuniform illumination for the antenna with a beamwidth $1.4^{\circ}$ is used. The quasi-periodic structure shows also a noticeable increase in the backscatter level at incident angles larger than $15^{\circ}$ and some variations in the peak values of the RCS along the Bragg directions. The effect of the surface irregularities was most evident from the backscatter measurement of power line \#4 as this cable has 21 outer aluminum strands that are loosely wound. Fig. 5(d) shows the measured and simulated $\sigma_{v v}$ of power line \#4 where the simulated results are those of a periodic structure with a nonuniform illumination similar to the incident field.

From the measured results it is also noticed that the increase in the level of backscatter at angles larger than $15^{\circ}$ is much higher for VV than it is for $\mathrm{HH}$. This is due to the fact that the vertical component of the electric field couples very well with the almost horizontal grooves and produces more backscatter, as shown in [11].

Another parameter of practical importance to be considered is the effect of power line sag on its backscatter response. A power line cable when suspended between two towers conforms to a curve known as catenary. The parameters of this function are determined by the physical parameters of the cable such as weight per unit length, the mechanical properties of the strands, the span of the supporting towers, and the environmental effects such as temperature and the existence of an external load such as ice. To simulate the effect of power line sag on its RCS, simulation of RCS of power line \#1 suspended between two steel towers $250 \mathrm{~m}$ apart at $-5^{\circ} \mathrm{C}$ with an ice load of $0.65 \mathrm{~kg} / \mathrm{m}$ is considered. This configuration generates a displacement of 


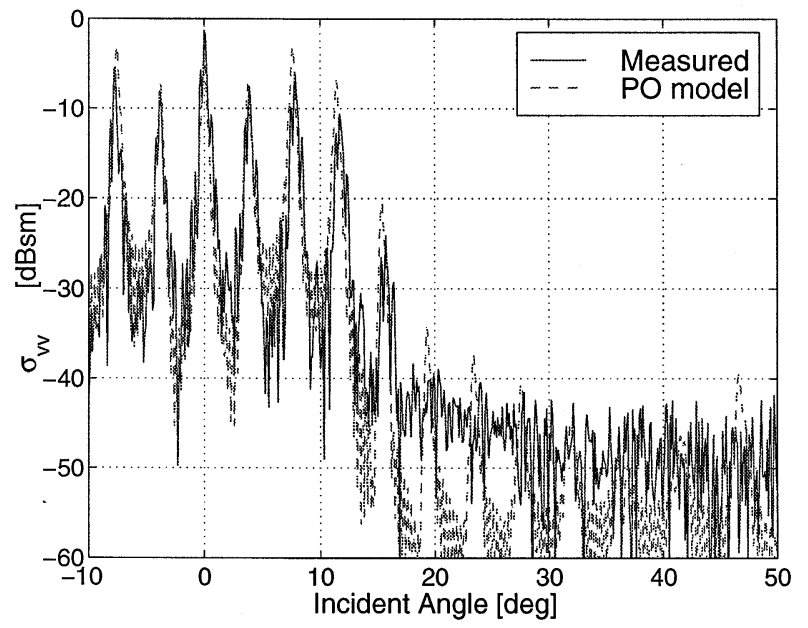

(a)

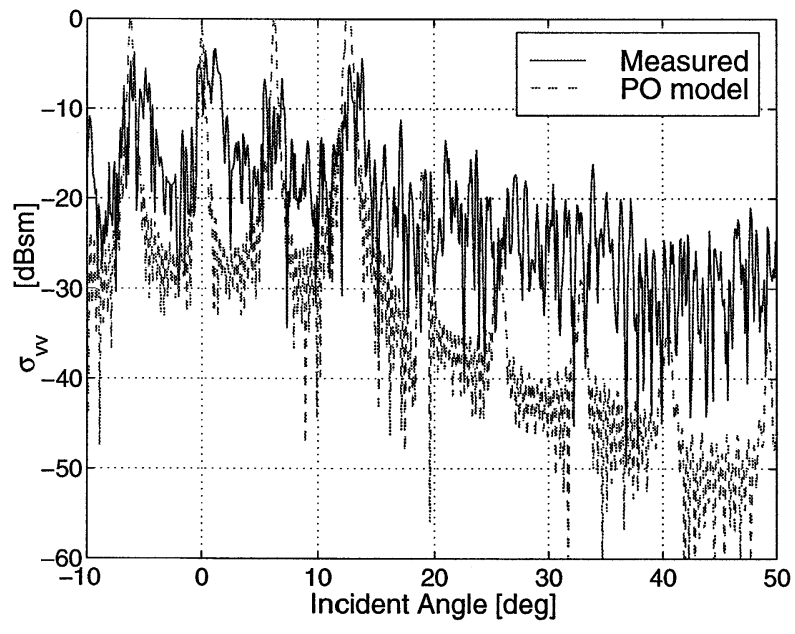

(c)

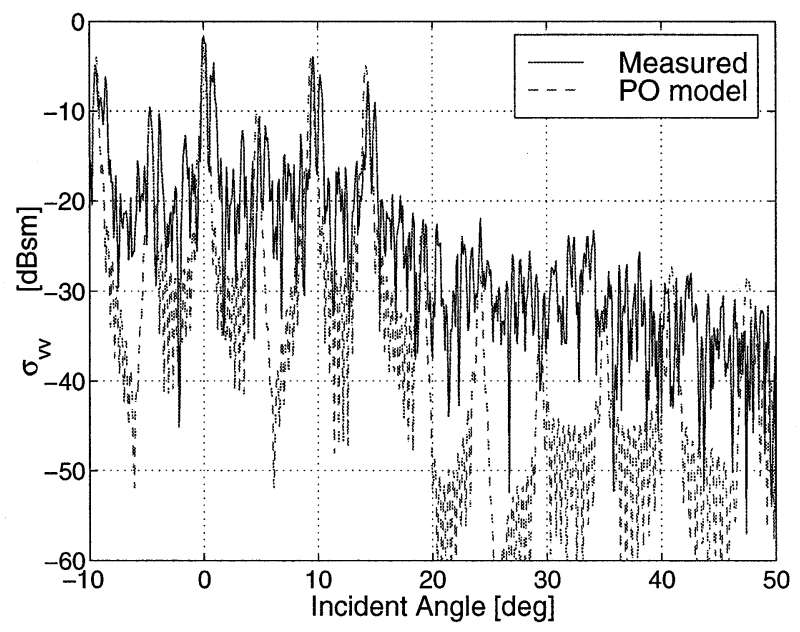

(b)

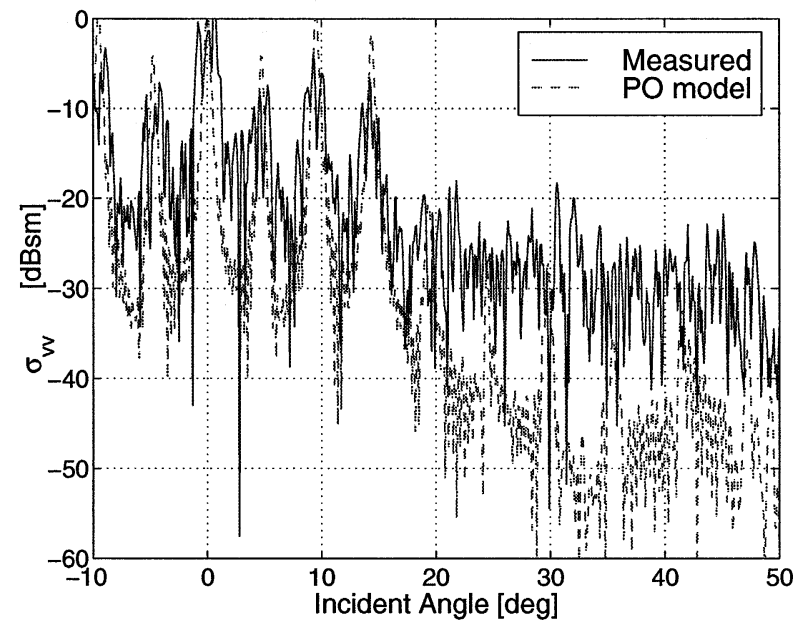

(d)

Fig. 5. Comparison of the simulated (assuming a Gaussian Beam) and measured VV polarized backscatter responses of the four power line samples used in this study: (a) power line \#1, (b) power line \#2, (c) power line \#3, (d) power line \#4.

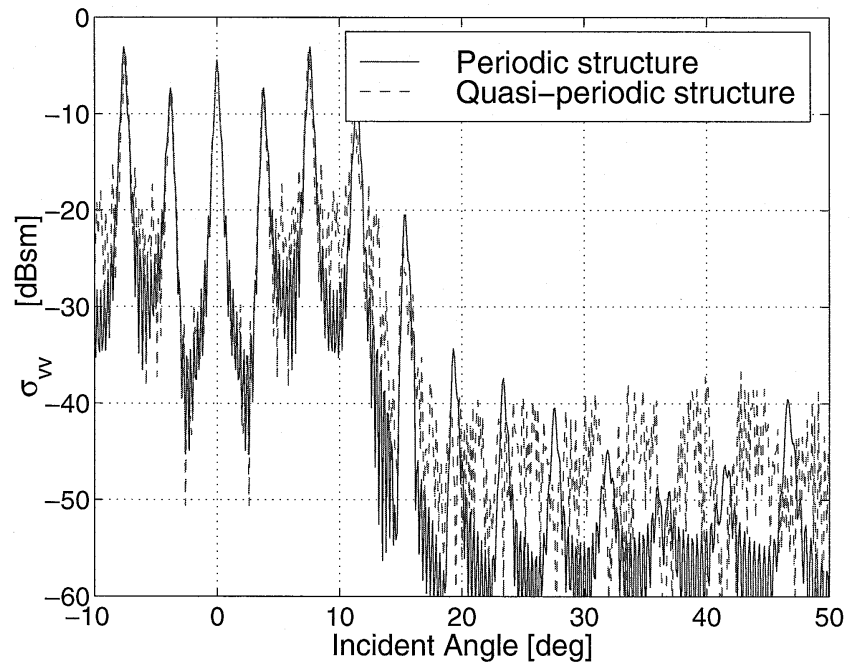

Fig. 6. Effect of surface irregularity for power line \#1. In this simulation the power line structure is modified by inserting gaps between adjacent strands. The size of the gaps are randomly distributed from zero to $1 / 20$ of the strand diameter.

about $6.6 \mathrm{~m}$ in the middle. The temperature and the ice load were selected to simulate deeper sag, which is expected to ex- hibit more influence on the power line RCS. Here a comparison is made between the backscatter response of a curved and a straight power line. RCS of the line is calculated for two different ranges, 100 and $500 \mathrm{~m}$, using a real beam antenna with beamwidth of $1.4^{\circ}$. The center of the radar beam is chosen at a distance $10 \mathrm{~m}$ from the left end of the cable. At normal incidence, the footprint sizes for 100 and $500 \mathrm{~m}$ are 2.4 and $12 \mathrm{~m}$, respectively. Fig. 7 shows the comparison between the RCS of a straight and a curved cable at 100 and $500 \mathrm{~m}$, respectively. The main effect of the sag on power line RCS is observable on the RCS level of Bragg backscatters at incidence angles lower than $17^{\circ}$. It is shown that a suspended cable has RCS values about 5 $\mathrm{dB}$ lower than that of a straight cable at normal incidence and at the third Bragg direction (about $8^{\circ}$ from normal incidence), and slightly higher values at other Bragg angles. Since there still exists strong backscatter for near-normal aspect angles, the detectability will not be affected much by the curvature of power lines.

It is worth mentioning that the effect of an ice layer on power lines cannot be easily modeled because of the existence of nonuniform icicles and ice thickness. However, in [2], the effect of a layer of ice on different power lines is characterized experimentally. 


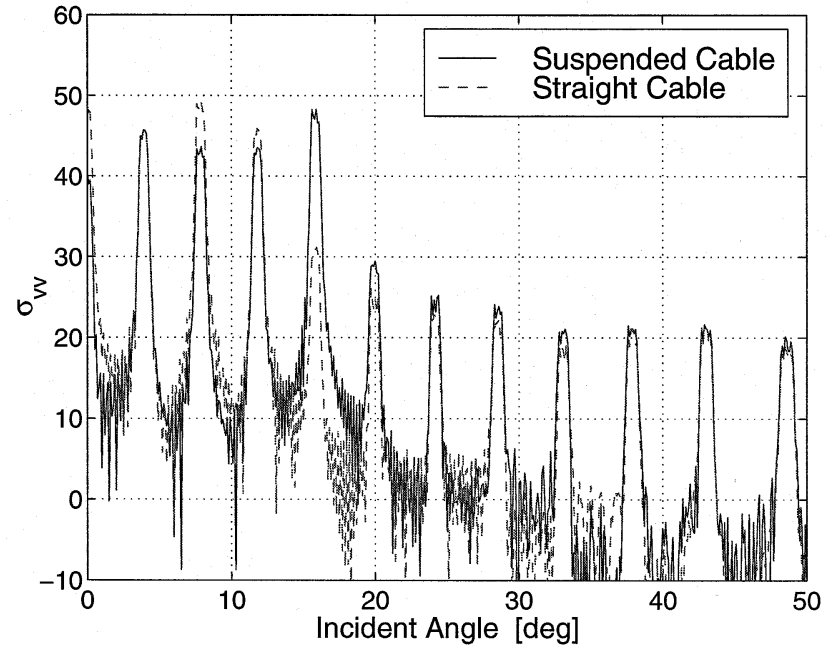

(a)

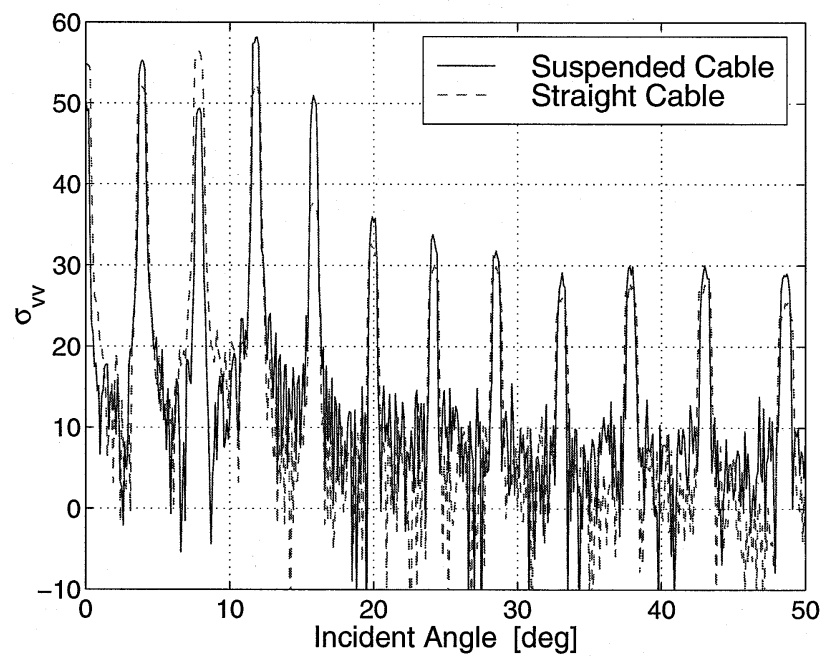

(b)

Fig. 7. Effect of sag on the radar backscattering response. Span $=250(\mathrm{~m})$, Sag $=6.6(\mathrm{~m})$, Radar beam $=1.4^{\circ}$. The center of the antenna beam is located $10 \mathrm{~m}$ away from the left-end of the cable. (a) Range $=100$ (m). (b) Range $=500(\mathrm{~m})$.

\section{CONCLUSION}

Electromagnetic scattering formulation for braided cables and power lines is formulated in this paper. Magnetic field integral equation in conjunction with periodic Green's function is developed as a basis for the calculation of the scattered field. This formulation together with the method of moment presents a viable approach for the field calculation at frequencies up to $K u$ band beyond which it becomes numerically inefficient. An approximate solution based on iterative physical optics approximation was proposed as an alternative for millimeter-wave frequencies. Through comparison with experimental data at 94 $\mathrm{GHz}$, it was shown that the iterative PO solution up to second order is capable of predicting the polarimetric backscatter responses of power line cables. This solution was used in a sensitivity analysis to study the effect of different geometrical parameters of power lines on their polarimetric backscatter response.

\section{REFERENCES}

[1] Aviation Week Space Technol., p. 103, July 6, 1987.
[2] K. Sarabandi and M. S. Park, "Millimeter-wave radar phenomenology of power lines and a polarimetric detection algorithm," IEEE Trans. Antennas Propagat., vol. 47, pp. 1807-1813, Dec. 1999.

[3] B. Rembold, H. G. Wippich, M. Bischoff, and W. F. X. Frank, "A MM-wave collision warning sensor for helicopters," in Proc. Military Microwave, 1982, pp. 344-351.

[4] H. H. Al-Khatib, "Laser and millimeter-wave backscatter of transmission cables," in Proc. SPIE Physics and Technology of Coherent Infrared Radar, vol. 300, 1981, pp. 212-229.

[5] M. Savan and D. N. Barr, Reflectance of wires and cables at $10.6 \mathrm{mi}-$ crometer, in Center for Night Vision and Electro-Optics, Jan 1988.

[6] K. Sarabandi and M. Park, "Extraction of power line maps from polarimetric SAR images," IEEE Trans. Antennas Propagat., vol. 48, pp. 1802-1809, Dec. 2000.

[7] K. Sarabandi, L. Pierce, Y. Oh, and F. T. Ulaby, "Power lines: radar measurements and detection algorithm for SAR images," IEEE Trans. Aerosp. Electron. Syst., vol. 30, pp. 632-648, Apr. 1994.

[8] F. Obellerio-Basteiro, J. L. Rodriguez, and J. R. Burkholder, "An iterative physical optics approach for analyzing the EM scattering by large, open-ended cavities," IEEE Trans. Antennas Propagat., vol. 43, pp. 356-361, Apr. 1995.

[9] K. Sarabandi and T. C. Chiu, "Optimum corner reflectors for calibration of imaging radar," IEEE Trans. Antennas Propagat., vol. 44, pp. 1348-1361, Oct. 1996.

[10] A. Nashashibi, K. Sarabandi, and F. T. Ulaby, "A calibration technique for polarimetric coherent-on-receive radar system," IEEE Trans. Antennas Propagat., vol. 43, pp. 396-404, Apr. 1995.

[11] T. B. A. Senior, K. Sarabandi, and J. Natzke, "Scattering by a narrow gap," IEEE Trans. Antennas Propagat., vol. 38, pp. 1102-1110, July 1990.

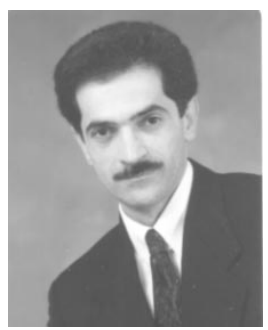

Kamal Sarabandi (S'87-M'90-SM'92-F'00) received the B.S. degree in electrical engineering from Sharif University of Technology, Tehran, Iran, in 1980. He received the M.S. degree in electrical engineering, the M.S. degree in mathematics, and the $\mathrm{Ph} . \mathrm{D}$. degree in electrical engineering from The University of Michigan, Ann Arbor, in 1989.

$\mathrm{He}$ is the Director of the Radiation Laboratory and a Professor in the Department of Electrical Engineering and Computer Science (EECS), University of Michigan. His research areas of interest include microwave and millimeter-wave radar remote sensing, electromagnetic wave propagation, and antenna miniaturization. He has 20 years of experience with wave propagation in random media, communication channel modeling, microwave sensors, and radar systems and is leading a large research group including four research scientists and ten Ph.D. and two M.S. students. Over the past ten years he has graduated $15 \mathrm{Ph} . \mathrm{D}$. students. He has served as the Principal Investigator on many projects sponsored by NASA, JPL, ARO, ONR, ARL, NSF, DARPA, and numerous industries. He has published many book chapters and more than 105 papers in refereed journals on electromagnetic scattering, random media modeling, wave propagation, antennas, microwave measurement techniques, radar calibration, inverse scattering problems, and microwave sensors. He has also had more than 220 papers and invited presentations in many national and international conferences and symposia on similar subjects.

Dr. Sarabandi is a Member of Commission F of URSI and of The Electromagnetic Academy. He is a Vice President of the IEEE Geoscience and Remote Sensing Society (GRSS), Chairman of the Awards Committee of the IEEE GRSS, and a Member of IEEE Technical Activities Board Awards Committee. $\mathrm{He}$ is the Associate Editor of the IEEE TRANSACTIONS ON ANTENNAS AND PROPAGATION and the IEEE SENSORS JOURNAL. He is listed in American Men \& Women of Science, Who's Who in America, and Who's Who in Electromagnetics. He received the Henry Russel Award from the Regent of The University of Michigan (the highest honor the University of Michigan bestows on a faculty member at the assistant or associate level). In 1999, he received a GAAC Distinguished Lecturer Award from the German Federal Ministry for Education, Science, and Technology given to about ten individuals worldwide in all areas of engineering, science, medicine, and law. He was also a recipient of a 1996 Teaching Excellence Award from the EECS Department of The University of Michigan. 
Moonsoo Park was born in Seoul, Korea, in February 1964. He received the B.S. and M.S. degrees in electronics engineering from Seoul National University in Seoul, Korea, in 1987 and 1989, respectively. He is pursuing the Ph.D. degree in electrical engineering at the University of Michigan, Ann Arbor.

From 1989 to 1993, he was an RF/Microwave Design Engineer with Daewoo Electronics Company, Seoul, South Korea. In November 2000, he joined Andrew Corporation and is currently a Senior RF Engineer, where he leads a team of RF/fiber optic engineers in the design and development of high-performance fiber optic distributed antenna systems for wireless in-building applications. His research interests include polarimetric millimeter-wave radar systems and for navigation, millimeter-wave remote sensing, and SAR image processing. 